\title{
Review: onset of schizophrenia before 22 years of age is associated with abnormal presentation at birth and complicated caesarean birth
}

\author{
Verdoux H, Geddes JR, Takei N, et al. Obstetric complications and age at onset in schizophrenia: an international collaborative \\ meta-analysis of individual patient data. Am J Psychiatry 1997 Sep;154:1220-7.
}

\section{Objective}

To examine, using meta-analysis, the relation between a history of obstetric complications and family history of schizophrenia, age at onset, and sex.

\section{Data sources}

Published case control studies were identified by a Medline search, by scanning the bibliographies of relevant papers and previous review articles, and by contacting researchers.

\section{Study selection}

Studies were selected if they had a case control study design that examined the association between obstetric complications and schizophrenia.

\section{Data extraction}

Published and unpublished individual data were collected on 15 specific obstetric complications rated by the scale of Lewis and Murray. Data, if available, were also extracted on sex, birth order, age at onset of schizophrenia, and family history of schizophrenia. Weighted average estimates were calculated using logistic regression.

\section{Main results}

Raw data was obtained on 854 patients with schizophrenia from 11 different research groups. A positive history of obstetric complications (definite or equivocal according to the Lewis and
Murray scale) was found for $408(48 \%)$ patients. Of the 716 patients for whom the differentiation between definite and equivocal obstetric complications was available, 227 (32\%) had a positive history for definite obstetric complications. An association was found between age of onset of schizophrenia $(<22 \mathrm{y} v$ $\geqslant 22 \mathrm{y}$ ) and a history of definite obstetric complications (crude odds ratio [OR] 1.52, 95\% CI 1.04 to 2.22). The crude OR indicates a $52 \%$ increase in definite obstetric complications among patients with schizophrenia with earlier age at onset compared with those with late onset. Patients with onset of schizophrenia before 22 years of age were 2.7 times more likely than those with onset at a later age to have had a history of breech or abnormal presentation at birth (weighted average OR 2.67, CI 1.01 to 7.04) and 10 times more likely to have a history of complicated or emergency caesarean birth (weighted average OR 10.05, CI 1.21 to 83.32). No association was found between obstetric complications and family history of schizophrenia or sex.

\section{Conclusion}

Onset of schizophrenia before the age of 22 years is associated with breech or abnormal presentation at birth and complicated or emergency caesarean birth.

Source of funding: in part, the Stanley Foundation.

For article reprint:Dr H Verdoux, Service Universitaire de Psychiatrie, Centre Carreire, 121 rue de la Béchade, 33076 Bordeaux Cedex, France. Fax +33556561733.

\section{Commentary}

The study by Verdoux et al is an essential addition to knowledge about the relation between obstetric complications and schizophrenia. There is a strong evidence base for genetic factors as risk factors for schizophrenia. ${ }^{1}$ Non-genetic risk factors are less clear. Although not all studies have found a link, ${ }^{23}$ a recent systematic review and meta-analysis by Geddes and Lawrie reported an association between obstetric complications and schizophrenia. ${ }^{4}$ This review by Verdoux et al extends the previous review by using individual patient data to clarify which particular types of obstetric complications account for the increased risk.

As we approach replicated gene linkages in schizophrenia, it is timely to be provided with a guide to the exploration of gene environment interactions. Genetic research has shown us that to find genes that account for a small percentage of heterogeneous disorder, we need to pool disparate studies to gain adequate power. Verdoux et al have applied similar principles to tease out the links between obstetric complications and schizophrenia.

As detailed by McGrath and Murray, however, "we know too little about the range of factors that can impact on the orderly development of the brain, and our current methods of assessing neurodevelopment are crude in the extreme." 5 The findings of Verdoux et al warrant serious inspection because despite the lack of precision in measurement, an effect emerged between obstetric complications and age of onset.

There are implications for research and the need to collaborate with perinatologists, and for more cross fertilisation between those interested in obstetric complications and schizophrenia and other neurodevelopmental diseases. Maybe, one day, we will be able to reduce some of these risk factors, and perhaps reduce the incidence of this important group of disorders.

Paul White, MB Walston Park Hospital Brisbane, Queensland, Australia

1 Mowry BJ, Nancarrow DJ, Levinson DF. The molecular genetics of schizophrenia: an up-
date. Aust N Z J Psychiatry 1997;31:704-13.

2 Done DJ, Johnstone EC, Frith CD, et al. Complications in pregnancy and delivery in relation to psychosis in adult life: data from the British perinatal mortality survey sample. BMJ 1991;302:1576-80.

3 Buka SL, Tsuang MT, Lipsitt LP. Pregnancy delivery complications and psychiatric diagnosis. A prospective study. Arch Gen Psychiatry

4 Geddes JR, Lawrie SM. Obstetric complications and schizophrenia: a meta-analysis. $\mathrm{BrJ}$ Psychiatry 1995;167:786-93.

5 McGrath J, Murray R. Risk factors for schizophrenia: from conception to birth. In: Hirsch SR, Weinberger DR, editors. Schizophrenia. Oxford: Blackwell Science, 1995 